\title{
Metamaterial computation and fabrication of auxetic patterns for architecture
}

\author{
- Roberto Naboni \\ ACTLAB - Politecnico di Milano, Department \\ $\mathrm{ABC}$, Italy \\ roberto.naboni@polimi.it \\ - Lorenzo Mirante \\ ACTLAB - Politecnico di Milano, Italy \\ lorenzo.mirante@mail.polimi.it
}

\begin{abstract}
The paper investigates the potential of auxetics in architectural applications by means of computational design and additive manufacturing. This class of metamaterials expresses interesting behaviour related to the unusual characteristics of a negative Poisson's ratio. Different patterns have been studied through a design workflow based on parametric software and the use of Particle Spring systems to support the form-finding process of bending-active auxetic structures. An advanced understanding of their bending capacity is explored with the use of variable infill patterns informed by structural analysis. Furthermore, principles for the design and fabrication of auxetic gridshells are discussed.
\end{abstract}

Keywords: Auxetics, Computational Design, Form-Finding, Synclastic Shell, 3D-printing

\section{Introduction}

Typically, the materials to which we are accustomed have positive Poisson's ratio, that is, the act of stretching is expected to cause shrinking and the act of compressing results in bulging. However, this common knowledge has been challenged by auxetics, a class of materials which exhibit the unusual property of becoming wider when stretched and narrower when compressed (Evans, 1991). This results from having a negative Poisson's ratio (v) - the ratio of the lateral contractile strain to the longitudinal tensile strain for a material undergoing tension in the longitudinal direction (Evans, 2000). This class of materials has a relatively recent history, even if they have been known among scientists for about a century. One of the earliest known publications on this topic is titled Foam Structure with a Negative Poisson's Ratio (R.S. Lakes, 1987), but the term auxetic first appeared in the scientific article Molecular Network Design (Evans

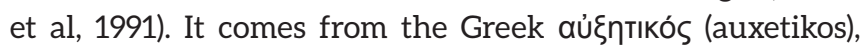
literally translated in "which tends to increase" and has its

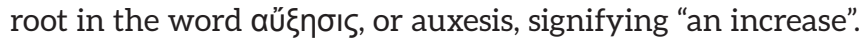
Auxetics can be essentially considered as metamaterials which are artificially engineered to gain emerging properties and functionalities otherwise unattainable in natural materials (Chaturvedi, 2009). They rely on specific spatial arrangements rather than material composition, and for this reason they are organized in patterns with precise shape, geometry, size, orientation and arrangements. Performance and behaviour are direct consequence of the design of their inherent architecture. Expanding this concept, the research investigates the architecture of auxetic materials towards their implementation in architectural structures, taking advantage of their unique properties.

\section{Mechanic features and current applications}

The deformation mechanisms of auxetics depend on their hinge-like structure, which flex outwards when stretched. Their spatial organization in particularly-shaped lowdensity patterns allows the hinge-like areas of the auxetic microstructures to flex. The study and the computational development of these patterns offer an interesting perspective for their future applications (Fig. 1). Auxetic structures exist in many different scales: from the microstructural and molecular to the mesoscopic and macroscopic scales (Evans, 2000). A large number of auxetic structures have been developed, such as foams, fibers, or composite materials, and many other examples can be found in nature as well (Liu, 2010). In this research the design and fabrication of macro-scale auxetics is explored for architectural applications. 

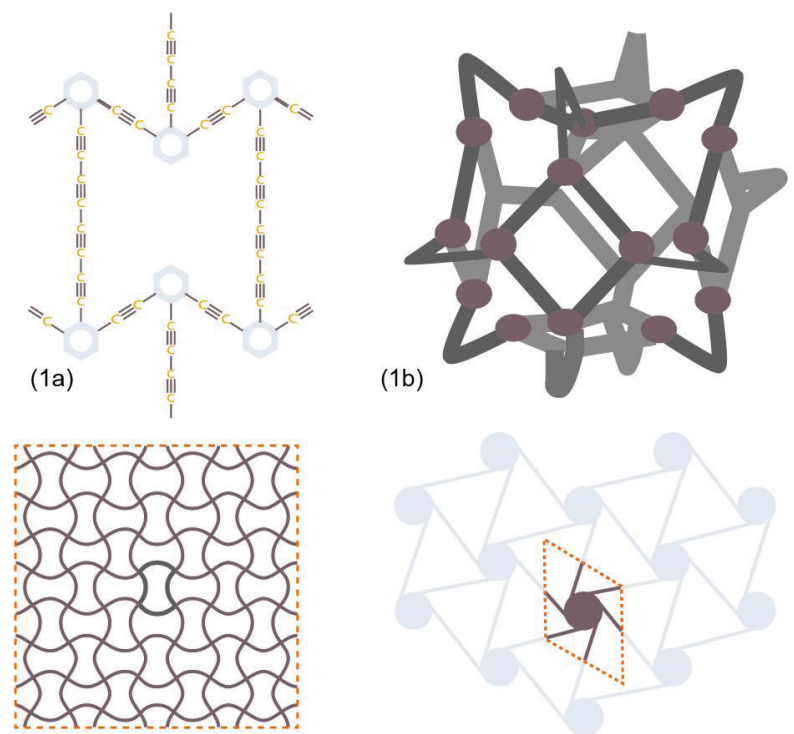

(1c)

(1d)

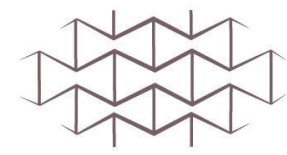

(1e)
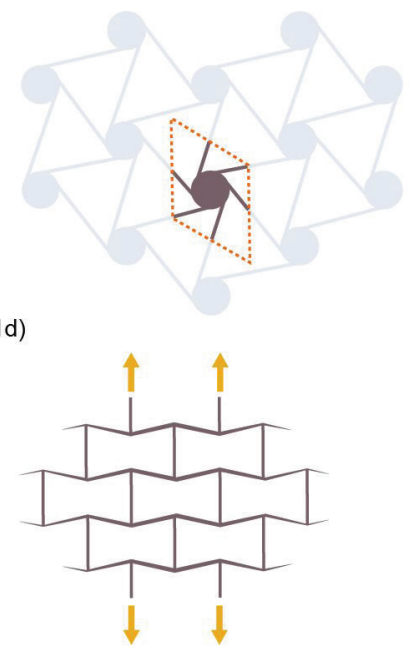

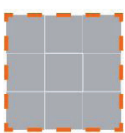

(1f)
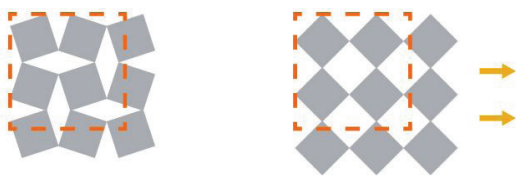

$\downarrow \downarrow$
Figure 1: Examples of auxetic structures. 1a) molecular auxetic, 1b) $3 \mathrm{~d}$ re-entrant, 1c) sinusoidal lattice, 1d) chiral, 1e) 2d re- entrant, 1f) square rotating rigid units.

Nowadays auxetic structures have found several applications in the biomedical industry (Scarpa, 2008) for the design of stents and prostheses (Abdelaal, 2008); in the creation of filters for chemical processes - auxetic foams mostly (Scarpa, 2015); in the generation of auxetic fibers for crash helmets/body armours (Sanami, 2014); as well as in the production of panels with high energy/vibration absorption coefficients (Yang, 2013). Interestingly, transforming a wellknown material into its auxetic configuration often offers improved mechanical features. Nevertheless, due to their low density and complex structure, auxetics are inefficiently manufactured with traditional processes, which involve complex multistep procedures with heat-compression molding. For this reason, current applications are generally restrained to high-tech fields. In medical and chemical sectors auxetics are mainly used for their capacity to variegate their porosity, whereas in medical, chemical engineering and in sport applications they are mainly exploited for their specific mechanical properties.

\section{Properties of auxetic structures}

The interest in auxetic structures experienced a remarkable spike during the last decades (Liu, 2006). A set of five fundamental characteristics of auxetic structures, actively explored and applied in various fields of research, can be outlined (Liu, 2010):

- Synclastic curvature: the capacity of auxetics to form dome-like, synclastic surfaces when bent (characterized by a positive Gaussian curvature $(\mathrm{K})$ at every point of the surface);

- Compressive strength and shear stiffness: the capacity of auxetic structures to resist forces both in compression and shear;

- Indentation resistance: the capacity of auxetic structures, mostly of foams, to shift more mass under the point of compression;

- Variable permeability: the capacity of auxetic structures to compress and expand, causing a variation in their porosity;

- Energy absorption and dissipation: the capacity of auxetic structures to absorb and dissipate the energy received from another body.

\section{Synclastic behavior}

Shear stiffness and synclastic curvature are the two fundamental features investigated within this research. Shear stiffness can be explained by the relationship between the Poisson's ratio $\mathrm{v}$ and the shear modulus $\mathrm{G}$ defined by Hooke's law, which states that when $v$ decreases to $-1, \mathrm{G}$ increases notably. In other words, the material becomes difficult to shear but easy to deform volumetrically (Liu, 2010). The second fundamental characteristic, regarding synclastic curvature, describes how planar auxetic structures tend to form a synclastic curvature when subjected to an out-of-plane bending moment - a behavior which is contrary to that of conventional elastic materials which display anticlastic curvatures when bent(Fig.2).The variation of a surface curvature is an effective parameter in defining different architectural results, and this research explores the computational form-finding of synclastic auxetic structures (Fig.3). 


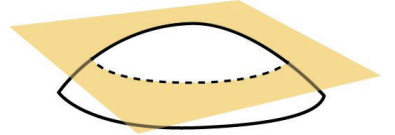

(2a)

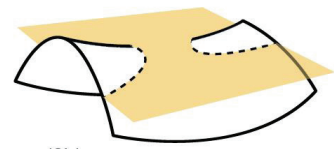

(2b)

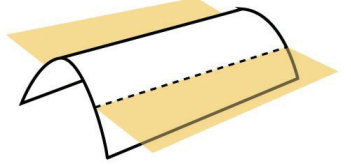

(2c)
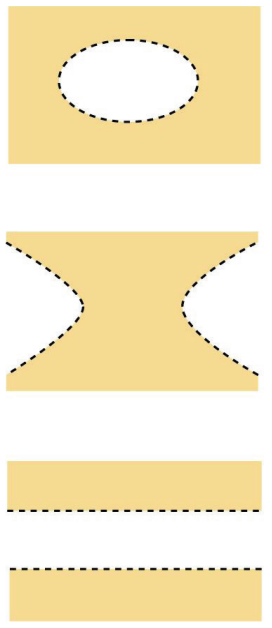

Figure 2: Typology of curvature 2a) Synclastic, 2b) Anticlastic and 2c) Developable.

Thecapacity of auxeticstogenerate synclastic curvatures suggests a development perspective as a bending-active structure which defines complex curved geometries from the erection processes of planar elements that are elastically deformed. This creates advantages in the transportation and assembly processes. The curved structures are influenced by residual stresses in their load bearing behaviour and structural capacities (Lienhard 2014). Moreover, working with sufficiently elastic structures, it is therefore possible to envision a dynamic process of shape adaptation through the implementation of kinetic structures.

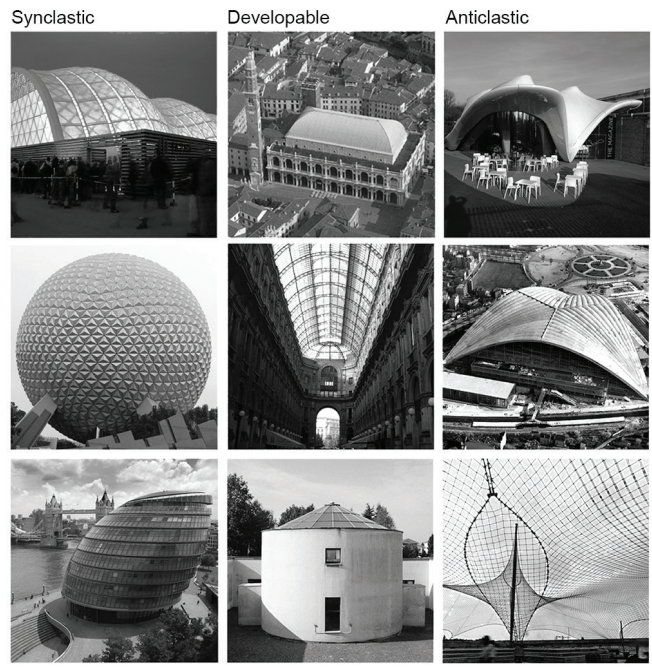

Figure 3: Architectural examples of different curvature typologies. In columns, from above: Synclastic: Shigeru Ban, Expo in Hannover, 2000 - Epcot Dome, Disney World, 1982 - Foster and Partners, London City Hall, 2002. Developable: Andrea Palladio, Basilica, Vicenza, 1614 - Giuseppe Mengoni, Galleria Vittorio Emanuele II, Milano, 1877 Aldo Rossi, Scuola, Fagnano Olona 1976. Anticlastic: Zaha Hadid, Pavilion for Serpentine Gallery, London, 2013 - Emmanuel Pouvreau, CNIT, Paris. 1958 - Frei Otto, Expo Montreal, 1967.

\section{Objective}

The objective of this research is to understand how auxetics can perform in architecture as bending-active structures towards the generation of lightweight synclastic gridshells. The generation of the form in this case might be directly driven and informed by the application of an out-ofplane bending moment and the physical behaviour of the auxetic material to which it responds. Synclastic surfaces in architecture are difficult to be achieved using traditional construction methods. Moreover, lightweight synclastic surfaces are currently limited to the use of air-supported structures.

Considering the necessity to preview the dynamic behaviour of bending-active structures, the research implements a computational design methodology to simulate the form-finding of synclastic auxetic gridshells. Additive Manufacturing (AM) is used in different phases to prototype test models as well as to empirically investigate different material configurations, to tune and enhance the response of an auxetic pattern. Sub-goals of the research are: (i) implementing a convenient computational methodology to design and simulate auxetic bending behaviour in a controlled way; (ii) determining optimal auxetic patterns for their structural implementation in architecture; (iii) understanding the main parameters which affect the configuration of auxetic structures and their spatial articulation; (iiii) proposing custom infill patterns to modulate bending performance in auxetic components.

\section{Methodological Procedures}

\section{Parametric design of auxetic patterns}

A variety of auxetic patterns exist and each of them is characterized by a set of parameters which influence their behaviour. In literature, auxetic structures are classified into several macro groups: re-entrant structures, chiral structures, rotating rigid units, angle-ply laminates, molecular auxetic structures, polymer models, origami-like structures and others (Mir, 2014). Scientific literature already tackled the topic of 3D origami-like auxetics and their potential applications in lightweight architectural structures (Schenk, 2010). Our research, on the other hand, focuses on twodimensional auxetics whose architectural potentialities have not been yet fully explored. In contrast to origami-like auxetics, these types of auxetic patterns achieve synclastic curvature through the active-bending of structural elements rather than through the rotation of faces along edges. In the initial phase of research, different basic patterns have been explored and developed parametrically with the use of Grasshopper for Rhinoceros as lattice samples divided into a defined grid of 20 by 20 cells. After the digital models were designed, prototypes were materialized with a double extruder FDM printer using a standard PLA filament allowing for an intuitive understanding of the bending properties of the different patterns (Fig. 4). 


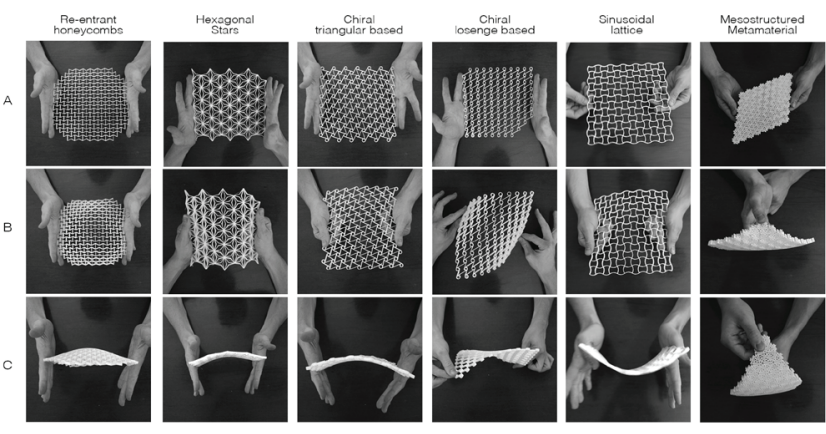

Figure 4: Comparative analysis on 3d printer auxetic patterns. Horizontal rows show: A) relaxed state - top view, B) compressed state - top view, C) compressed state - lateral view. All the samples perform auxetic behaviour in the in-plane stretching, while only the Re-entrant honeycombs and mesostructured patterns show auxetic response also in the out-of-plane bending.

The comparative tests highlighted the $2 \mathrm{D}$ re-entrant honeycombs as a compelling design option for further research and development given the enhanced synclastic curvature they are able to generate, their simple geometric configuration (Malgorzata, 2009) and ease of customization. This pattern is composed of indented elastic rods which are called Chevron Rods and inelastic rods which are called Parallel Rods (Fig. 5).

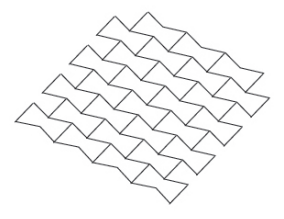

complete pattern

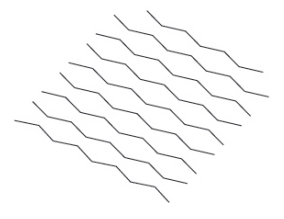

chevron rods

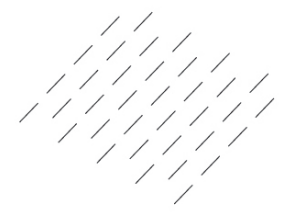

parallel rods
Figure 5:Schematization of rod typologies. There-entranthoneycombs pattern is defined by two types of rods: the indented elastic rods called the Chevron rods and the inelastic rods which are known as the parallel rods.

This bi-directional pattern reaches a different configuration according to the axial direction of bending forces (Fig. 6). Research and experiments on the creation of isotropic auxetic lattices can be found in literature (Lorato, 2010). In our research differential responses according to the bending direction is considered as an exploitable feature to generate less predictable architectural results. Empirical tests proved that forces applied on the indented sides produced synclastic curvatures in opposition with forces applied in the parallel direction.

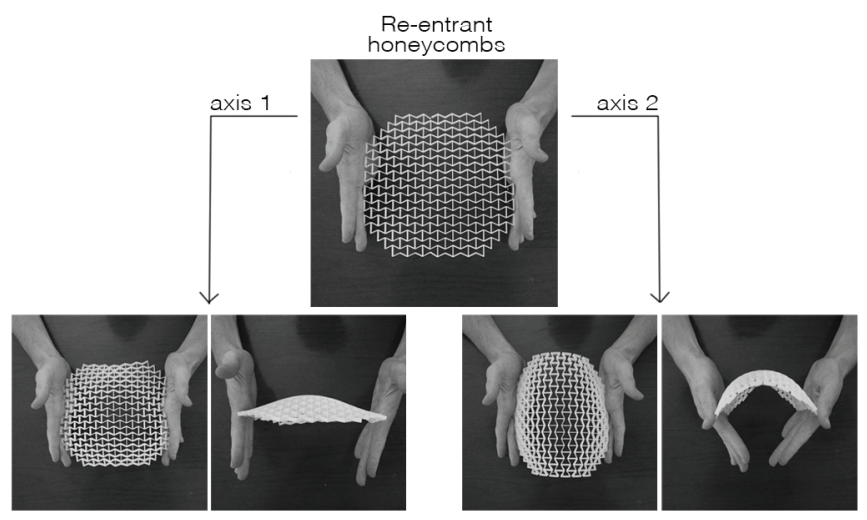

Figure 6: Bending analysis on a 3d printed model showing two different curvatures obtained by changing the axis of action.

\section{Auxetic behaviour in two dimensional patterns}

The simulation of the bending behaviour of an auxetic pattern is fundamental in order to develop its architectural application and to preview configurations under certain loading conditions. Considering the advantage of working within a common modelling environment, simulations were performed with the Particle Springs Engine (PS) Kangaroo for Grasshopper. This workflow guarantees the easy and effective testing of different pattern solutions by defining anchor points and forces applied to the structure, without the need of exporting geometries, a factor of great importance in evaluating many different patterns.

An initial test was performed on a default 2D reentrant honeycomb structure with no extrusion on the $\mathrm{z}$ axis in order to understand the auxetic expansion/ compression in relation to the variation of the parameter $\mathrm{t}$, which defines the angles characterizing the hexagons (Fig. 7a). Through the variation of this parameter within a range from 0 to 1 , it is possible to generate different kinds of hexagons: values from 0 to 0.5 define convex hexagons (Fig.7d), while values ranging from $>0.5$ to 1 produce re-entrant hexagons (Fig.7b and Fig.7c). The simulation shows how the variation of this parameter influences the Poisson ratio and consequently the auxetic properties of the structure. Fig. 7 shows the results of this test, with Fig.7b presenting the layout of the structure in a relaxed condition, Fig.7c showing the expanding behaviour of the structure in an auxetic configuration ( $t=0.73$ ), whereas Fig. $7 d$ reveals how the structure is stretched when turned into its non-auxetic version $(t=0.28)$ and the overall area decreases. 


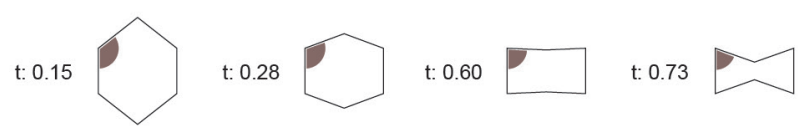

(7a)

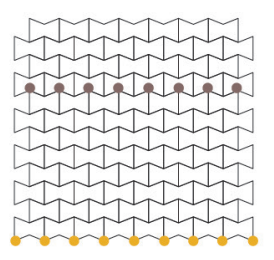

(7b)

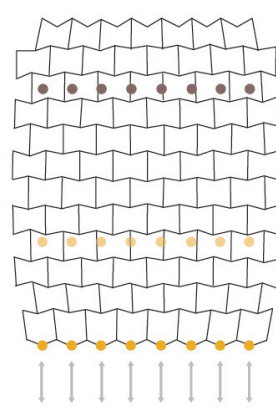

(7c)

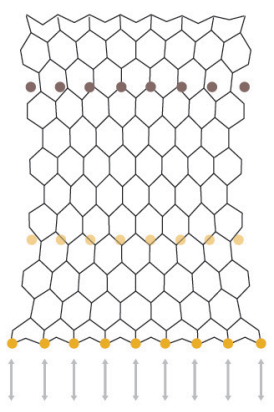

(7d)
Figure 7: The scheme shows how the change of the angular parameter t affects geometry and expansion geometry (7a). 7b) auxetic structure in relaxed state, 7c) auxetic structure in extension and 7d) non-auxetic structure in extension.

A subsequent test was carried out in a more complex situation where a homogeneous field of upward forces was applied to simulate out-of-plane bending. In opposition to the expected results, the simplified out-of-plane bending for an auxetic pattern ( $t>0,5)$ resulted in a developable surface instead of a synclastic one. This is to be understood as a consequence of testing patterns with no extrusion in the $\mathrm{z}$ axis, which are unable to provide sufficient sectional resistance to produce a synclastic curvature.

\section{Form-finding of form-active auxetic structures}

Subsequent tests were performed on patterns extruded in the $\mathrm{z}$-direction, converted into meshes and tested in Kangaroo, where physical forces were assigned to simulate the actual physical response of the prototyped patterns. A first set of forces (i) named as springs is used to keep both chevron and parallel rods stiff (Fig.8a), a second set (ii) of the same topology of forces is applied to guarantee the overall structure in tension (Fig.8b). A third set of forces (iii) is applied on two external edges of the structure to induce a moment of forces by the combination of linear vectors with opposite direction acting with equal distance from a pivot point (Fig.8d). Within internal and external loading configurations, a realistic bending behaviour is obtained by assigning the maximum stiffness parameters to all of the internal springs (i and ii), thus accurately reproducing the behaviour recorded in the physical prototypes.

Several experiments testing the influence of variable sectional heights of the rods have also been performed (Fig.9). With an extrusion of 1 unit the structure is not able to define a stable shell: the chevron rods are not rigid enough to support the stresses. Increasing the extrusion values up to 30 units, equivalent to $15 \%$ of the span, confers higher rigidity. (8a)
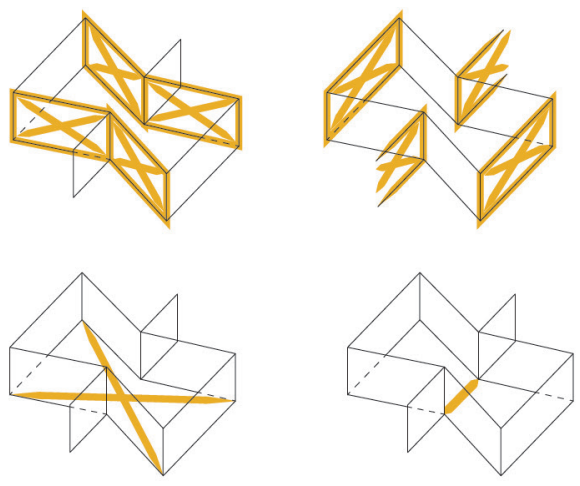

(8b)

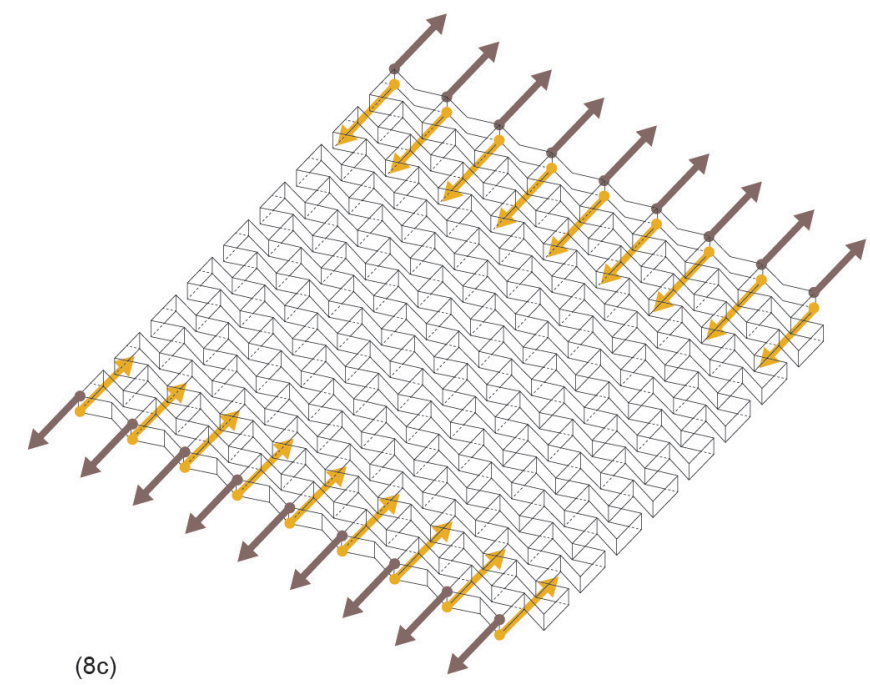

(8d)

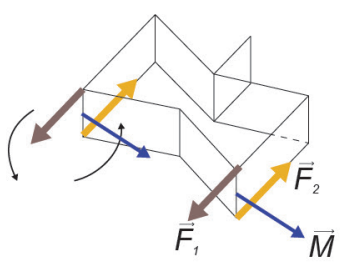

Figure 8: Forces set-up in Kangaroo. In 8a and 8b setting of internal springs; $8 \mathrm{c}$ represents general scheme of applied compression and tension forces; $8 d$ displays the moment of forces applied on the external edges.

Values ranging from $2,5 \%$ to $10 \%$ of the span of the shell are considered suitable for a bending-active structure. Variation of the parameter $t$ in structures with constant extrusion values determine the creation of synclastic and anticlastic shells, with values $t=0.76$ and $t=0.40$, respectively. Finally various patterns with variable extrusions were generated by a tonal image sampler to understand the influence of complex height arrangements in the form-finding process (Fig.10). 


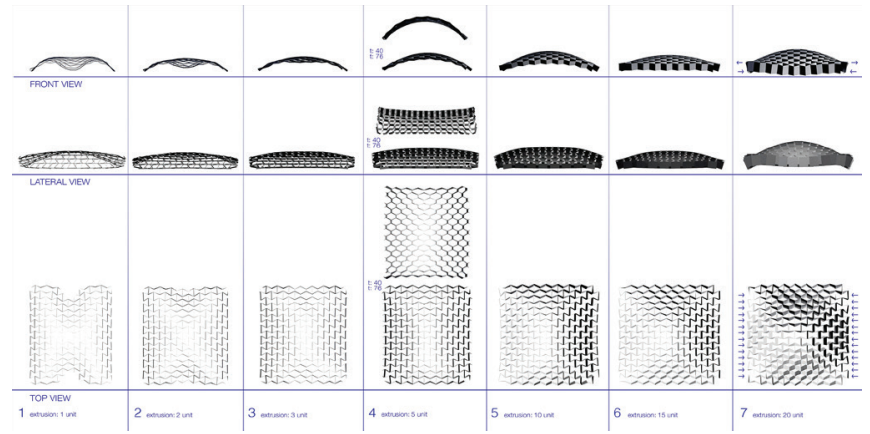

Figure 9: Test of the structure reaction to bending when thickness variations are applied.

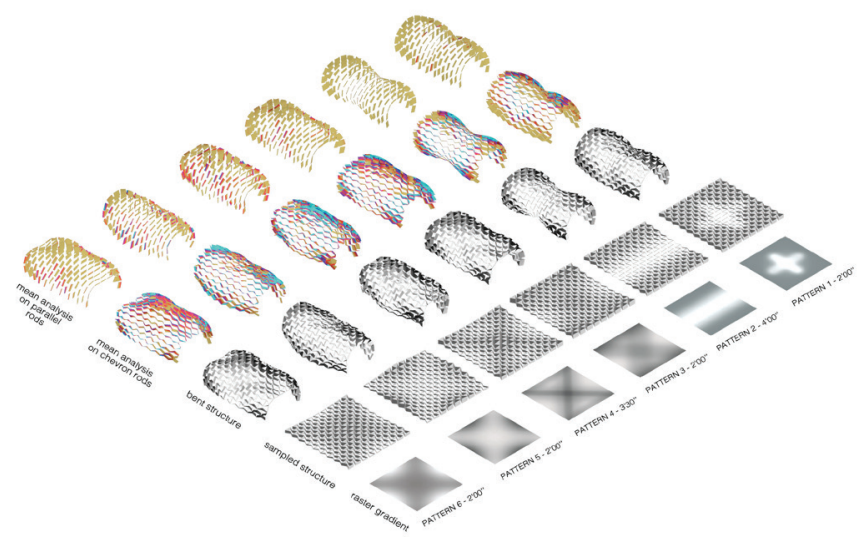

Figure 10: Differentiation of the pattern extrusion depending on the set of gradients. For each example is shown: flat configuration, bent configuration, curvature analysis of chevron rods and curvature analysis of parallel rods.

\section{Shape variations and microstructural optimization}

A further implementation of the form-finding process is possible through the description of the different initial grid shapes, which consequently inform different curvatures of the actively-bent structure. Changes in the initial configuration allows for the possibility to customize the structural morphology in order to produce articulated spatial compositions while reducing the number of anchor points needed on the ground. The resulting shapes were evaluated with the Finite Element (FE) solver of Millipede for Grasshopper which displays stresses and previews zones with excessive deflection levels. This structural analysis provides feedback to inform the extrusion values in any point of the shell, optimizing structural performance and material usage. Moreover, the Finite Element solver revealed that the active-bending produced and internalized a different amount of stress in each rod. This analysis can be mapped to optimize bending capacity along the structure and control deformation and resistance of the shell at any individual point. Initial experiments in the material organization of the auxetic structure are conducted by the typology and density variation of a simple infill grid: more dense infill is placed where higher rigidity is required, whereas less dense infill is used for the rods where an elastic behaviour is required in order to guarantee the active bending of the overall structure (Fig. 11).

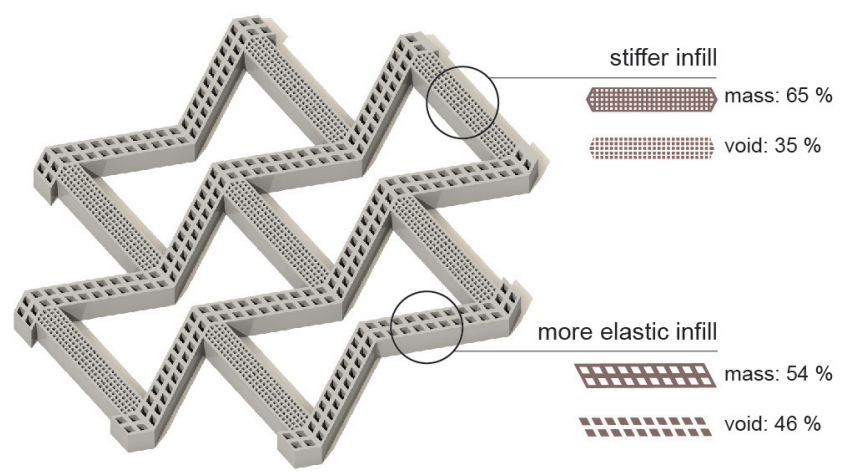

Figure 11: Infill with modulated stiffness for 3D Printing. More elastic infill is applied to the chevron rods to favour elasticity while the parallel rods are designed with stiffer infill.

\section{Results}

The design methodology described above produces various results in terms of computational workflow, understanding the bending-active behaviour of auxetics and in terms of fabrication processes.

\section{Computational workflow for designing auxetics}

An effective computational workflow within Grasshopper for Rhino for designing auxetic patterns and simulating their behaviour when subjected to forces is developed. The parametric definition of auxetic patterns allows the possibility to easily control features of the structure as well as differentiation of the module according to variable surface/ grid inputs. The Particle Spring (PS) system of the Kangaroo engine is used to preview the capacity to enlarge an area when pulled in two opposite directions and to produce synclastic geometries when bent. The research succeeded in approximating real bending forces and accurately reproducing the behaviour of physically printed prototypes. The possibility to simulate and tune the parametric model in the same design environment facilitates the iterative process of design.

\section{Pattern comparison}

Through this computational approach, a set of 3D-printed samples have been designed and tested showing typical inplane auxetic behaviour. However, in contrast to what was expected, only a few have produced synclastic curvatures when an out-of-plane bending moment was applied. Among the tested typologies, only the re-entrant honeycombs and 
mesostructured materials demonstrated an actual synclastic curvature. A comparative test highlights the 2D re-entrant honeycombs as a compelling design option for further research and development given the enhanced synclastic curvature they are able to generate, their simple geometric configuration (Malgorzata, 2009) and ease of customization towards the design of form-active gridshells.

Bending-active gridshells are essentially generated by the torsion of the elastic chevron rods whereas inelastic parallel rods ensure stability to the structure. The rigidity of the parallel rods and the flexibility of the chevron rods are the parameter that mostly characterize $2 \mathrm{D}$ re-entrant auxetic structures. Auxetic patterns were also discovered to have a specific axis which results in a synclastic curvature, in the case of $2 \mathrm{D}$ re-entrant lattice this corresponds to the direction perpendicular to the chevron rods.

\section{Parameters and pattern tuning}

Auxetic patterns can be tuned in their response through the modification of simple morphological parameters which determine the material response of the structure. Variable extrusion height and thickness contribute to modulate the resistance to torsion, which influences the shapeconfiguration of the gridshell. Differential infill patterns within the linear elements, which compose an auxetic cell, can be set to vary the behaviour of the chevron and parallel rods (see Fig. 10c). In particular, variations in the pattern topology affect the bending behaviour more so than variations in density. An interesting comparison can be made with the work of Schumacher (2015) in which three-dimensional figures are subdivided into different areas and - depending on the degree of stiffness/elasticity that is needed - are filled with microstructures that once printed will confer specific elastic behaviours to each area.

\section{Discussion}

Auxetic metamaterials have unique characteristics which are explored in their architectural potential by parametric modelling and Particle Spring systems. A workflow for the form-finding and analysis of bending-active structures based on auxetic patterns is proposed and effectively help to preview the bending behaviours of such structures. After an analysis of different options, the paper focuses on the study of $2 \mathrm{D}$ re-entrant structures which produce variably curved shells by the parametrization of a few parameters of the planar patterns. Their capacity to bend and support different loads is fundamental for architectural applications. The research identifies Additive Manufacturing (AM) as the ideal production process to control the implications of an optimized microstructural organization in order to tune the response of auxetic mega-structures. Future investigations will implement the discovered geometric and topological parameters into the creation of complex spatial arrangements at architectural scale.

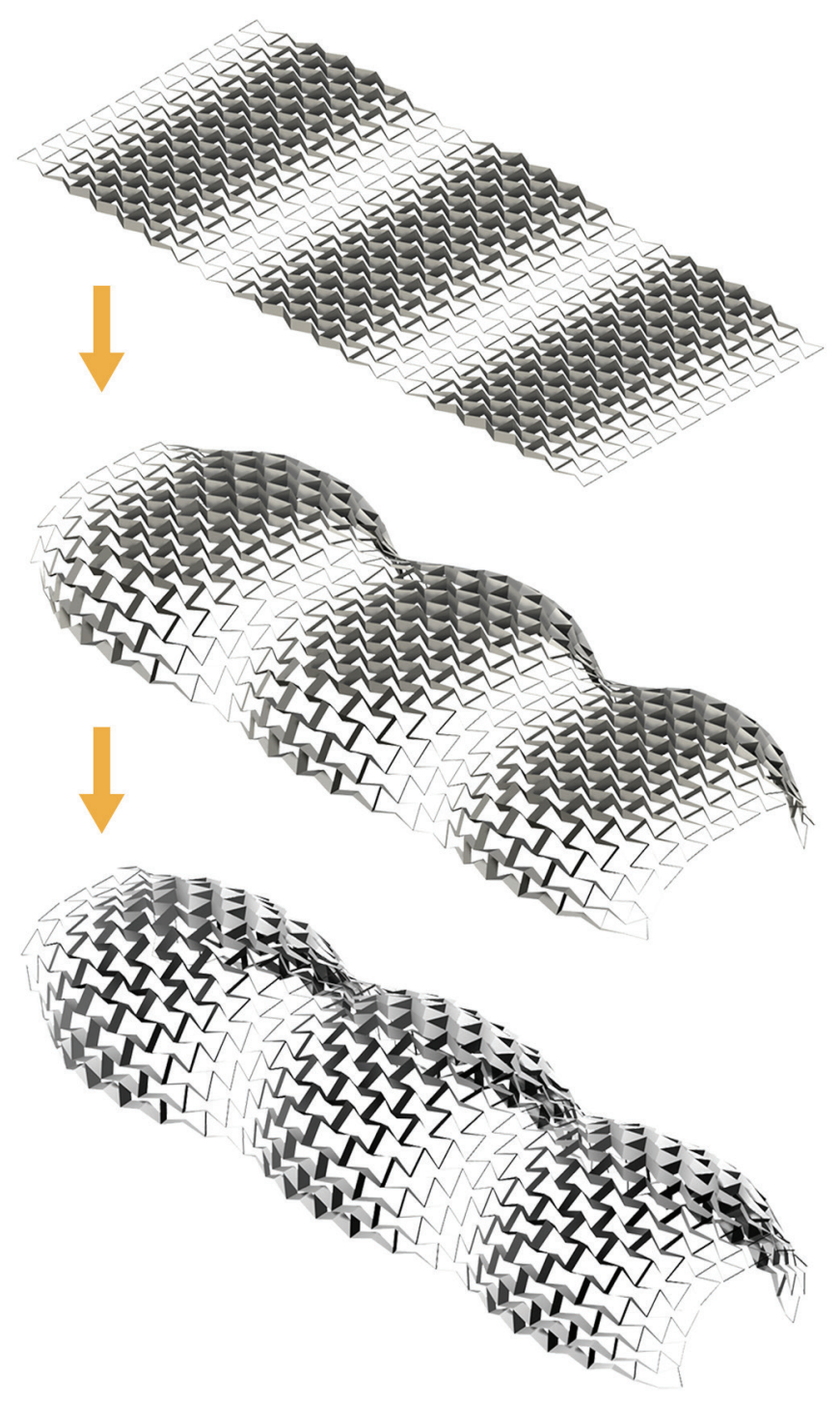

Figure 12: from top: planar structure, halfway bent structure, bent structure.

\section{Acknowledgements}

We would like to thank Prof. Ingrid Paoletti and our colleagues from ACTLAB for supporting this project with enthusiasm. We also express our gratitude to KLONER 3D which provided the fundamental fabrication equipment to develop this work.

\section{References}

Abdelaal, O. A. M., Darwish, S. M. H. (2008). Analysis, Fabrication and a Biomedical Application of Auxetic Cellular Structures. International Journal of Engineering and Innovative Technology, Volume 2, Issue 3, 218-223.

Chaturvedi, P. (2009). Optical Metamaterials: Design, Characterisation and Applications. University of Illinois at Urbana-Champaign. (Electronic Thesis or Dissertation). Retrieved from: http://web.mit.edu/nanophotonics/projects/ 
Dissertation_Pratik.pdf

Cremers, J. M. (2011). Energy Saving Design Of Membrane Building Envelopes. Structural Membranes 2011, 147-157.

Evans, K.E., Nkansah, M. A., Hutchinson, I.J., Rogers, S.C. (1991). Molecular Network Design. Nature, 353, 124.

Evans, K.E., Alderson, A. (2000). Auxetic Materials: Functional Materials and Structures from Lateral Thinking!. Advanced Materials, 12, No.9, 617-628.

Findley, T. (2013). An Experimental Analysis of Auxetic Folded Cores for Sandwich Structures Based on Origami Tessellations. (Electronic Thesis or Dissertation). Retrieved from: https://etd.ohiolink.edu/

Lakes, R. (1987). Foam structure with a negative Poisson's Ratio. Science, 235, 1038-1040.

Lienhard J. (2014). Bending-Active Structures. Form-finding strategies using elastic deformation in static and kinetic systems and the structural potentials therein. Institute of Building Structures and Structural Design, Stuttgart (Electronic Thesis or Dissertation). Retrieved from:

http://elib.unistuttgart.de/opus/volltexte/2014/9483/pdf/

Diss_Bending_Active_Structures_Julian_Lienhard.pdf

Liu, Q. (2006). Literature Review: Materials with Negative Poisson's Ratios and Potential Applications to Aerospace and Defence. (Electronic Thesis or Dissertation). Retrieved from: http://dspace.dsto.defence.gov.au/dspace/handle/1947/4436 Liu, Y., Hu, H. (2010). A Review on Auxetic Structures and Polymeric Materials. Scientific Research and Essays, 5(10), 1052-1063.

Lorato, A., Innocenti, P., Scarpa, C., Alderson, A., Alderson, K.L., Zied, K.M., Ravirala, N., Miller, W., Smith, C.W., Evans, K.E. (2010). The transverse elastic properties of chiral honeycombs. Composites, science and Technology, 70, 1057-1063.

Małgorzata, J-M. (2009). Micromechanical Model of Auxetic Cellular Materials. Journal of Theoretical and Applied Mechanics 47, 4, 1-14.

Mir, M., Murtaza, N.A., Javaria, S., Ansari, U. (2014). Review of Mechanics and Applications of Auxetic Structures. Advances in Materials Science and Engineering Volume 2014. Retrieved from: http://dx.doi.org/10.1155/2014/753496.

Mozafar, S.R., Yunan, P., Zaini, A. (2014). Analytical solution and finite element approach to the $3 \mathrm{~d}$ re-entrant structures of auxetic materials. Mechanics of Materials, 74, 76-87.

Otto F. and Burkhardt B. (1978). Multihalle Mannheim. Stuttgart: Freunde und Förderer der Leichtbauforschung Sanami, M., Raviralaa, N., Kim, A., Alderson, A. (2014). Auxetic Materials for Sport Applications. Procedia Engineering, 72, $453-458$.

Scarpa, F. (2008). Auxetic Materials for Bio Prostheses. IEEE Signal Processing Magazine, 126-128.

Scarpa, F. L., Giacomin, J. A., Bezazi, A., \& Bullough, W. A. (2006). Dynamic behavior and damping capacity of auxetic foam pads. In Proceedings of SPIE, San Diego CA, USA.

Schenk, M., Simon, D., (2010). Origami Folding: A Structural Engineering Approach. Presented at the 5OSME, 5th international conference on Origami in Science, Mathematics and Education. Retrieved from: http://www.markschenk. com/research/\#papers.

Schumacher, C. Bernd, B., Jan, R., Steve, M., Chiara, D., Markus, G. (2015). Microstructures to Control Elasticity in 3D Printing. Retrieved from:

http://www.disneyresearch.com/wp-content/uploads/ Microstructures-to-Control-Elasticity-in-3D-Printing-Paper. pdf

Seffen, K.A. (2012). Compliant shell mechanisms. Philosophical Transactions of the Royal Society A, 370, 2010-2026.

Theodoros, T. (2014). Modelling, Simulation and Verification of Pneumatically Actuated Auxetic Systems. Open Systems: Proceedings of the 18th International Conference on Computer-Aided Architectural Design Research, 395-404.

Tomohiro, T. (2013). Freeform Origami Tessellations By Generalizing Resch's Patterns. Journal of Mechanical Design. Yang, L., Harrysson, O., Cormier, D., West, H., Park, C., \& Peters, K. (2013). Design of Auxetic Sandwich Panels for Structural Applications. Solid Freeform Fabrication Symposium, 929. 